\title{
How much aerosol reaches the lungs of wheezy infants and toddlers?
}

\author{
B Salmon, N M Wilson, M Silverman
}

\begin{abstract}
Using sodium cromoglycate as a non-toxic marker, we assessed the delivery of aerosol to nine wheezy infants and seven healthy adults. The drug was delivered to the infants by face mask and spacer device from a metered dose inhaler, and by face mask and nebuliser to the infants and the adults who breathed though their noses. The concentration of sodium cromoglycate was estimated in timed urine collections to find out how much of the dose was absorbed from the lung. Only $0 \cdot 13 \%$ to $0.61 \%$ of the $20 \mathrm{mg}$ nominal dose was detected in the urine, representing an estimated $0.3 \%$ to $1.5 \%$ deposited in the lung. Nasal filtration of aerosol could be a critical factor in reducing lung deposition.
\end{abstract}

Many therapeutic trials of inhaled drugs for the treatment of asthma in wheezy infants and toddlers have failed to show any appreciable beneficial effects. An essential step in understanding why this is so, is to find out if enough drug reaches the target organ. In adults under optimum conditions only about $14 \%$ of an inhaled dose reaches the lungs. ${ }^{12}$ Most studies entail inhalation of a radiolabelled marker, or require invasive procedures such as serial venepunctures. Because of this, objective information on pulmonary drug deposition in young children has not been available.

Assay of sodium cromoglycate in plasma has been used as a means of assessing inhaled drug delivery in adults. It is safe and free from side effects. Its pharmacokinetics after inhaled, intravenous, and oral administration have been investigated extensively in adults. ${ }^{23}$ It is water soluble and remains in the extracellular compartment, predominantly in the ionised form at physiological pH. Sodium cromoglycate is absorbed efficiently from the respiratory tract. In contrast, less than $1 \%$ of an oral dose is absorbed from the gastrointestinal tract. Once absorbed it is not metabolised and is rapidly cleared from plasma. There are two routes of excretion: renal and biliary. Quantitatively they are of roughly equal importance, $43 \%$ of the absorbed dose being excreted in the urine. Radioimmunoassay of the unchanged drug in plasma and urine can be done. ${ }^{4}$

In a small pilot study we established that after treatment of young children we could detect sodium cromoglycate in urine. There are no data on its pharmacokinetics in young children.

The aim of this study was to assess the amount of drug deposited in the respiratory tract of young children using urinary excretion of sodium cromoglycate as the marker. We compared two methods of administration-jet nebuliser and metered dose inhaler with a valved spacer device - and compared the results in children with an assessment of the respiratory deposition of sodium cromoglycate in seven adults using the same jet nebuliser and methods. The latter comparison allowed us to test the assumption that the pharmacokinetics of sodium cromoglycate were similar in infants and adults. We did not use the face mask and valved spacer in the adults because this method of administering the aerosol is not commonly used in that age group.

\section{Subjects and methods}

We studied nine boys aged between 9 and 36 months (median 16). All had frequent and recurrent wheezy episodes but were well when they attended the outpatient department on the two study days. Boys were chosen because it was easier to collect urine samples from them.

On one study day the treatment was by an Acorn nebuliser (Medic Aid) driven by compressed air at $8 \mathrm{l} /$ minute. Sodium cromoglycate respirator solution $(20 \mathrm{mg}$ diluted to $4 \mathrm{ml}$ with normal saline) was nebulised for 10 minutes and administered during tidal breathing using a Hudson oxygen facemask (Henleys Medical Supplies) held against the face. On the other study day the same dose of sodium cromoglycate (four puffs of Intal-5) was given from a metered dose inhaler into a multispacer (Medic Aid). This has lightweight inspiratory and expiratory valves, which are protected by a shield from clogging up with powder. The proximal end was attached to a facemask with a cushioned rim (Medic Aid) and applied firmly around the nose and mouth to achieve an airtight seal. The infant took several breaths from the spacer for each actuation of the metered dose inhaler.

The order of administration was random. As soon as control urine samples had been obtained treatment was given. Timed serial urine samples were collected into 24 hour collecting bags, which were checked at frequent intervals and drained as soon as the parent or investigator noted that urine had been passed. Urine volumes were recorded. Leakage was measured by recording the change in weight of the preweighed nappy.

A single study was carried out using the Acorn nebuliser and facemask in seven healthy adults. Using the same dose and protocol, they inhaled sodium cromoglycate and collected urine samples at $1,2,3,4,6$, and 8 hours. They
Correspondence to: Dr Silverman.

Accepted 4 December 1989 
Cumulative urinary excretion of sodium cromoglycate at four hours

\begin{tabular}{llllll}
\hline & \multicolumn{1}{c}{ Children } & & \multicolumn{2}{c}{ Adults } \\
\cline { 2 - 5 } & Spacer & $p$ Value & Nebuliser & p Value & Nebuliser \\
\hline $\begin{array}{l}\text { Median }(\mu \mathrm{g}) \\
\begin{array}{l}\text { Mange } \\
\text { Median percentage } 20 \mathrm{mg} \text { nominal } \\
\text { dose }\end{array}\end{array}$ & 26 & $<0.05$ & 66 & $<0.05$ & 130 \\
\hline
\end{tabular}

were instructed to use tidal nasal breathing from the facemask.

An aliquot of each urine sample was frozen. Analysis was carried out on coded samples by radioimmunoassay, the technician being unaware of when the specimen had been taken.

\section{ANALYSIS}

Obviously the infants did not void to a time schedule and to compare subjects it was necessary to interpolate a value for a given arbitrary time point. Cumulative urinary excretion of sodium cromoglycate was plotted against time. Most curves were approaching a plateau by four hours, so this time was taken. For each subject, data were plotted in two ways: firstly, they were fitted to a rectangular hyperbola assuming first order pharmacokinetics for excretion of sodium cromoglycate, ${ }^{3}$ and secondly they were plotted by joining adjacent time points but making no assumption about drug kinetics. There was excellent agreement between the methods. The values reported here are based on the first method. Wilcoxon's rank sum test was used to compare the two modes of administration of sodium cromoglycate for children, and the Mann-Whitney $U$ test for comparison of results of data from adults and children.

\section{Results}

Analysis of cumulative excretion curves indicated that over $90 \%$ of the final total had been excreted by four hours in both infants and adults.

The cumulative urinary excretion of sodium cromoglycate at four hours was significantly greater when the children were treated with nebuliser than with a spacer $(p<0.05)$. After nebulisation the urinary excretion was significantly greater in adults than in children $(p<0.05)$ (table). The amount excreted was a minute proportion of the nominally delivered dose. There were wide individual differences: one child and one adult given nebulised sodium cromoglycate excreted more than double the maximum of the other subjects in their group. We learned afterwards that the adult concerned had in fact breathed orally during the study instead of, as instructed, breathing nasally. We can only speculate on the chosen route of inhalation of the infants, but suspect that as they were settled and cooperative they breathed nasally most of the time.

\section{Discussion}

Sodium cromoglycate is efficiently absorbed from the respiratory tract, but only $0.8 \%$ of an oral dose is absorbed from the gastrointestinal tract. $^{23}$ The area under the curve for plasma concentrations of sodium cromoglycate after inhalation correlates well with its urinary clearance, and plasma concentrations increase with doses within the range $2-20 \mathrm{mg} .^{5}$ Its action is not related to its plasma concentrations, which is an indication of the amount absorbed and thus indirectly of the amount actually delivered to the respiratory tract.

In adults $43 \%$ of an intravenous dose of sodium cromoglycate is excreted unchanged in the urine within 90 minutes. ${ }^{2}$ In our study of seven healthy adults breathing through their noses we calculated that only $1.5 \%$ of $20 \mathrm{mg}$ of sodium cromoglycate was absorbed after delivery by nebuliser and face mask. This lends support to the argument that the delivery system is a limiting factor in the efficient delivery of the drug to infants.

Although there are no data concerning the pharmacokinetics of sodium cromoglycate in young children, there is no theoretical reason why it should differ from adults. If we speculate that the urine:plasma ratio is the same in young children as it is in adults we can calculate that for the nebuliser $0.76 \%$ of the nominal dose of $20 \mathrm{mg}$ of sodium cromoglycate was absorbed. In proportion to body size this represents a larger effective lung dose than for adults. For the spacer, the equivalent value was $0 \cdot 30 \%$. Even if only $70 \%$ of the drug that was deposited on the respiratory mucosa was absorbed, ${ }^{3}$ it is clear that delivery of sodium cromoglycate to the respiratory tract was low whichever method of inhalation was used.

A proportion of the nebulised dose must have been swallowed. Even if only $0.8 \%$ of this were absorbed, the quantity could have contributed appreciably to the sum excreted, leading to an overestimate of the dose absorbed from the respiratory tract. Thus the true dose delivered may have been even lower.

How much can we generalise from data collected using one specific jet nebuliser and one particular valved spacer? The Acorn nebuliser, run at $8 \mathrm{l} /$ minute, has an output of 0.34 $\mathrm{ml} /$ minute and a mass median aerodynamic diameter of 4.5 microns (D Cameron, M Clay, personal observations). These figures are close to the ideal for therapeutic aerosols. The spacer was probably less than ideal. Its output was only $27 \%$ of that of a more widely used device (Nebuhaler, Astra) when they were assessed in vitro using a multistage impinger with a flow rate of $60 \mathrm{l} /$ minute (A Clark, personal observations). This is, however, greater than the flow rate that a toddler will generate under tidal breathing conditions. Using a more efficient spacer, the lung dose absorbed after delivery by 
metered dose inhaler and spacer might have been greater. Our choice of spacer was determined by the fact that the Medic Aid spacer has lighweight inspiratory and expiratory valves, and has an easily attachable airtight face mask. The use of a sealing facemask attached to a Nebuhaler (Astra) with the inspiratory valve held open by gravity had been described for delivery for aerosols to infants. ${ }^{6}$ The amount of drug reaching the lungs using this device has not, however, been reported.

Obviously there is wastage of the drug; a residue is left in the nebuliser or spacer and some escapes into the atmosphere during expiration. We attempted to measure the former but our measurements were unreliable, because a surfactant in the propellant interfered with the drug assay.

If sodium cromoglycate can be absorbed from the nasal mucosa in infants breathing nasally, our data will have overestimated pulmonary deposition of sodium cromoglycate. Adults were asked to breathe through their noses to mimic the infants' pattern. Interestingly, the one subject who did not do so, and breathed through his mouth instead, had the highest value for urinary excretion of sodium cromoglycate. The estimated lung dose for that subject was $4.4 \%$ of the nominal dose. It seems likely that the nose acts as a filter, reducing pulmonary deposition to about a quarter of the value obtained during mouth breathing.

We conclude that sodium cromoglycate is a useful and safe marker of respiratory tract deposition in children and that it can be used to compare the efficacy of different drug delivery systems. Though the nebuliser may have delivered more drug than the spacer and metered dose inhaler, the clinical importance of the difference is not clear. Toddlers and their parents seem to prefer the spacer to the more time consuming nebuliser. Delivery by either nebuliser or metered dose inhaler with spacer gave poor pulmonary deposition. It is therefore possible that some of the problems of treatment in young children are the result of inadequate amounts of the drug reaching the lungs. Improved techniques for drug delivery are needed to overcome the barrier imposed by the nose.

We thank Action Reserch for the Crippled Child and the Asthma Research Council for their support. Fisons plc kindly analysed the urine sam les.

1 Newman SP, Pavia D, Garland N, Clarke SW. Effects of various inhalation modes on the deposition of radioactive pressurised aerosols. Eur $\mathcal{f}$ Respir Dis 1982;63(suppl 119):57-65.

2 Neale MG, Brown K, Hodder RW, Auty RM. The pharmacokinetics of sodium cromoglycate in man after intravenous and inhalation administration. Br $\mathcal{F}$ Clin Pharmacol 1986;22:373-82.

3 Richards R, Dickson CR, Renwick AG, Lewis RA, Holgate ST. Absorption and disposition kinetics of cromolyn sodium and the influence of inhalation technique. $\mathcal{F}$ Pharsodium and the influence of inhalation
macol Exp Ther 1987;241:1028-32.

4 Brown K, Gardner JJ, Lockley WJS, Preston JR, Wilkinson DJ. Radioimmunoassay of sodium cromoglycate in human plasma. Ann Clin Biochem 1983;20:31-6.

5 Patel KR, Tullett WM, Neale MG, Wall RT, Tan KM Plasma concentrations of sodium cromoglycate given by nebulisation and metered dose inhalers in patients with exercise-induced asthma: relationship to protective effect. Br f Clin Pharmacol 1986;21:231-3.

6 O'Callaghan C, Milner AD, Swarbrick A. Spacer device with face mask attachment for giving bronchodilators to infants with asthma. Br Med 7 1989; 298:160-1. 